\title{
Neurosurgeons, Acute Care Surgeons or Moms: Who Should Care for the Head Injured?
}

\section{Thomas J. Esposito*}

Division of Trauma, Surgical Critical Care and Burns, Department of Surgery, Loyola University Medical Center, Maywood, Illinois, USA

\section{Preface}

Traumatic brain injury is a significant problem in American health care which taps a tremendous number of resources [1]. Neurosurgeons are an integral part of head injury care along with the trauma surgeon, particularly in those cases involving multi system trauma. The intensely trained neurosurgical practitioner dedicated to the care of a broad range of neurologically based conditions, including trauma, is in short supply. Furthermore, like their general surgical colleagues, they are being taxed not only by the glut of head injuries but also by the attendant social, financial and perceived legal disincentives associated with their care. That is why, together, we must find a way to share and reduce the burden of head injury care for both practitioner types and keep both engaged in this vitally needed service to society.

It is toward that end that this admittedly provocative and "tongue-in-cheek" essay is offered. Its purpose is not to single out neurosurgeons for castigation, but rather, the intent is to stimulate spirited, yet collegial, honest and productive debate of fundamental issues. It is crucial that these issues be resolved expeditiously in order to move forward and provide much needed access to quality care that is rendered by well trained, committed practitioners who draw pride and satisfaction from their work.

When I was a kid and fell hitting my head, or was hit in the head with a baseball or "got my bell rung" playing football, it didn't automatically generate an ED visit, or a trauma team activation, a neurosurgery consult, one or several CT scans (or skull x-rays since CT didn't exist), an MRI or even a hospital, much less an ICU, admission. When I was a kid, there was no propensity to file a malpractice suit and no paranoia on the part of doctors fearing litigation. There was no malpractice premium crisis or concern for reimbursement. When I was a kid and I or one of my peers sustained a head injury in the neighborhood, there was mostly just a mom who provided observational care, diligently waking us up from our sleep every two hours until morning. My how things have changed - except, perhaps, the largely observational nature of head injury care!

A recent study presented a profile of patients with head injuries enrolled in the National Trauma Data Bank (NTDB') highlighting the nature of their injuries and care [2]. This showed that nearly $95 \%$ of patients received observational or non-interventional care with only $4 \%$ requiring craniotomy and $2 \%$ undergoing ICP monitoring. Of those undergoing craniotomy, the median time to get to the OR was 3 hours and 15 minutes with only $6.5 \%$ of those craniotomies performed within one hour of admission.

It would seem rational to propose that the only time you really need a "brain surgeon" is to do "brain surgery." Observational care does not seem to fall under the category of "brain surgery". There is no contention that a neurosurgeon should also be intimately involved with the decision of who, in fact, needs or may need brain surgery. However this involvement does not necessarily mandate the immediate physical presence of that neurosurgeon in this era of telemedicine and teleradiography. Furthermore, it appears craniotomy, when it does occur, happens at more than 3 hours after admission. This is certainly within most EMS and trauma systems' capabilities of patient transfer and transport to facilities where neurosurgeons committed to trauma care are available to perform surgery.

Regardless of the actual need for services exclusively and uniquely provided by a board certified neurosurgeon, one cannot begin to explore the issue at hand without considering supply and demand factors.
It is estimated that 1.5 million Americans sustain traumatic brain injuries annually [3]. The demographics of practicing neurosurgeons (particularly in rural areas) precludes all of these patients being seen by a neurosurgeon currently, and perhaps to an even lesser degree in the future [4]. This is much the same as the unreasonable expectation that every patient with abdominal pain presenting to the ED be seen by a general surgeon.

In addition to a numerical undersupply of neurosurgeons to meet the need, there also exists a functional lack of neurosurgeons. Many practicing neurosurgeons have recently chosen to eliminate trauma care from their practice and/or relinquish craniotomy privileges at their hospitals. Unpublished data from the Society of Neurological Surgeons Liability Insurance Task Force [5] shows that $75 \%$ of academic neurosurgery departments responding to a survey reported that private neurosurgeons in their communities were doing less intracranial work and $88 \%$ reported private neurosurgeons doing less trauma call. This same study showed that $79 \%$ of responding departments have experienced an increase in referrals as a result of changing practices of private neurosurgeons.

Corroborating these findings, and the suggestion that all may not be well with neurosurgical emergency care, are federal data from the GAO showing that $42 \%$ of hospitals reported problems with neurosurgical on-call coverage. This places the specialty second on a list of twelve.

*Corresponding author: Thomas J. Esposito MD, MPH, Department of Surgery, Loyola University Medical Center, 2160 S. First Avenue, Building 110, Room 3276, Maywood, IL 60153, USA, Tel: 708- 327-2072; Fax: 708-327-3474; E-mail: tesposi@ lumc.edu

Received April 23, 2012; Accepted June 04, 2012; Published June 06, 2012

Citation: Esposito TJ (2012) Neurosurgeons, Acute Care Surgeons or Moms: Who Should Care for the Head Injured? J Trauma Treat 1:137. doi:10.4172/2167 1222.1000137

Copyright: (c) 2012 Esposito TJ. This is an open-access article distributed under the terms of the Creative Commons Attribution License, which permits unrestricted use, distribution, and reproduction in any medium, provided the original author and source are credited. 
Fourteen percent of hospitals report similar problems with general surgery which ranks eighth on that same list of twelve [6]. There are concrete examples of this from the Neurosurgical literature as well [7].

Results from yet another study show that at least $20 \%$ of neurosurgeons prefer not to treat trauma patients and more would give up taking emergency department call were it not mandated in order to maintain hospital privileges [8]. While this is a lesser degree of reticence to treat trauma patients than expressed by general surgeons, the smaller pool of neurosurgeons available magnifies the impact of any degree of reduction in neurosurgical services on access to care.

Hard evidence from evaluation of system operations supplement these survey data in pointing to a concerning trend. A study of state trauma system transfer patterns has revealed a $44 \%$ increase in transfers of head injured patients over a recent five year period in the face of only a $14 \%$ increase in head injured patients entered into the state trauma registry over the same time interval [9]. Additionally, the mean injury severity score (ISS) of these patients was less than 16 (the generally accepted threshold of severity considered to warrant treatment at a trauma center). The majority of these interfacility transfers were from Level II trauma centers, where neurosurgical services should theoretically be available, to Level I trauma centers. Other data from neurosurgeons in Illinois, support these findings regarding transfers $[10,11]$. These studies describe the negative impact of transfers on academic medical centers, neurosurgical training, financial viability and, most concerningly, patient outcome.

Contributing to the exodus of neurosurgeons as well as other surgical specialists from participation in trauma care are the perceptions of increased medicolegal risk, disproportionate reimbursement with regard to work effort in comparison to elective cases and spine work, and increased liability insurance premium rates attached to the inclusion trauma care within the scope of practice. Finally, lifestyle issues play a role as well. This physical and functional undersupply of surgeons, even at tertiary centers, has created a void in care of patients presenting to Emergency Departments which is approaching crisis proportion $[12,13]$.

The purpose of this dissertation is not to target and castigate neurosurgeons. Similar situations, unfortunately, exist in other specialties, including general surgery and orthopedics, when it comes to emergency care. Neurosurgery issues, however, have precipitously risen to the forefront as the most notable, perhaps serving as the proverbial canary in the coal mine.

Who, then, shall fill any voids in neurotrauma care that exist? It has been proposed from the realm of trauma surgery that an Acute Care Surgeon (ACS), broadly trained and appropriately credentialed, can do so $[14,4]$. This has met with swift and resounding opposition from the leadership of neurosurgery $[15,16]$. But on what foundation?

The first supposition would most logically be that this is a reflex defensive posture to a perceived "turf" transgression. It is true that the majority of "academic" trauma surgeons as well as "rank and file" general surgeons (60\% and $49 \%$ respectively) favor including limited neurosurgical procedures in the scope of practice for Acute Care Surgeons[11,17]. However when examined more closely, respondents to these surveys ranked the addition of select and limited neurosurgical procedures last on a list of eleven features seen as enhancing their current practice $[17,18]$. This would seem to temper any interpretation of a desire to actively and aggressively transgress neurosurgical "turf". Alternatively, it seems to convey an attitude of Trauma or Acute Care
Surgeons wanting to be ready, willing, and able, to provide appropriate head injury management in the interest of good patient care when a fully trained neurosurgeon is not available, for whatever reason. This is substantiated by Valadka's [19]. Survey of surgeons on issues pertaining to neurotrauma. His conclusions were that the trauma surgery community would welcome the support and the assumption of significant responsibility for neurotrauma care which is perceived to be lacking.

There may be a disconnect between the neurosurgical leadership and their constituents practicing (or, perhaps, not fully practicing) in the community. The data cited earlier, and more data from the neurosurgical literature, suggests the rank and file neurosurgeons are voting with their feet $[20,10,9,8,6,13,11,5,19,7]$. Valadka [19] has shown that only $32 \%$ of trauma surgeons he surveyed indicated that neurosurgeons were in charge of head injury care if no operation had been performed, and only $60 \%$ reported that neurosurgeons were in charge of adult patients with isolated head injuries who had been operated upon. He has also reported on this same lack of presence in the Emergency Department for all types of neurosurgical emergencies [7].

One has to assume that the neurosurgical leadership would desire Valadka's survey numbers to be closer to $100 \%$. Critics can argue that these are only perceptions from an opinion survey, but one can conversely argue that perception is reality. Be it perception or reality, the message being sent seems to be that community neurosurgeons are disengaged and disenchanted with care of the neurotrauma patient and emergency call of any type.

This is much different than what organized neurosurgery would lead us to believe. On the receiving end, overtaxed neurosurgeons at level I trauma centers may welcome relief from other providers, who can share the increasing work load generated by unnecessary transfers, reduce the number of night calls, and the need to perform some procedures. This may be especially true in centers without residents or even at centers with neurosurgical training programs in this era of limited resident work hours $[20,11]$. Many are now reverting to reliance on neurologists or other non-neurosurgeons and even non-physicians such as physician assistants and nurse practitioners to share this burden with little publicized objection by the neurosurgical leadership.

Some neurosurgeons have recently cited unpublished data to suggest that over $90 \%$ of neurosurgeons responding to a survey report "participating" in their hospital's call schedule [16]. Although hard to reconcile with previously cited evidence suggesting otherwise $[10,9,6,13,11,5]$, these findings are potentially believable given the fact that some neurosurgeons reportedly demand and receive, on the average, stipends as high as several thousand dollars per day for "participating" [21]. However, this seems to be a craftily worded revelation that is difficult to interpret, much less accept, without the definition of "participate." "Taking call" and "taking care of" or "taking responsibility for" are different things. Taking call for spine but not intracranial cases, taking call for non-trauma cases but not trauma cases, or taking call for trauma (sometimes for a stipend) and then transferring the patient to another facility (sometimes without personally examining the patient) are not uncommon practices witnessed in the community today.

Liberal and expeditious transfer to regionalized neurotrauma centers seems to be a strategy toward which a number of neurosurgical leaders are leaning as a solution to access issues [15,16]. This is based on Norwegian data showing better outcomes for patients who 
were transferred to the care of neurosurgeons at a specialized center rather than cared for locally by non-neurosurgeons [22]. However, in contradistinction, work done in the rural environment of this country does demonstrate benefit to decompressive procedures performed by properly trained non neurosurgeons at local hospitals when timely access into a neurosurgeon is not possible [23]. In that analysis, Rinker reported from rural Montana that 7 of 8 patients meeting criteria for local decompression of subdural or epidural hematomas survived and were functioning independently at a mean follow-up interval of 4 years.

On closer scrutiny of the Norwegian study, overall injury severity and measures of acute head injury severity were not collected or compared between groups. Glasgow outcome scores were reported and found to be worse for the patients operated on at the outlying hospital. More than likely this was due to the greater number of subdural hematomas operated on at that hospital. The median time to craniotomy was shorter in the outlying hospital than at the regional center ( $6.5 \mathrm{hrs}$. vs $12 \mathrm{hrs}$.). The authors themselves admit this speaks to the sicker patients, not able to tolerate transfer and who are more prone to have poor outcomes, being operated on at the outlying hospital. They discuss the possibility of a "selection mechanism" or sample bias as a plausible alternative explanation of results rather than a difference in surgical expertise.

For the sake of argument, one would have to question the training and experience of those Norwegian non-neurosurgeons performing decompressive surgery in comparison to what is proposed for the Acute Care Surgeon of the future in the U.S. Subsequent critical care was also not controlled for. Finally, these European findings may not hold true for non-operative management.

An interesting criticism of Rinker's study [23] and an explanation for its more positive results put forth by neurosurgeons is that it involved significant participation by members of their specialty in the training and support of the non-neurosurgeons. But this is exactly what the proponents of Acute Care Surgery are seeking! [19]

Regardless of whether expeditiously transferring head injured patients to regionalized specialty centers garners better outcomes than local management, liberal transfers can overburden level I centers. Transports within any time frame, particularly if they are unnecessary, are not without their financial and other costs, not the least of which is adverse effect on patient outcome $[20,10,11,5]$. One can envision a role for an Acute Care Surgeon in obviating some of these transfers at the local level, if allowed to treat these patients locally. Conversely, the Acute Care Surgeon might serve to lessen the burden on neurosurgeons at tertiary referral centers.

Frankly, there is little perceived enthusiasm on the part of current general trauma surgeons for performing formal craniotomies. So few patients undergo craniotomy, it is hardly worth making this a major point of contention between the disciplines. Placement of ICP monitors and non-operative management may be a different matter, however.

It would appear that abdication of critical care of the head injured patient is already taking place [24]. The utilization of neurologists, or intensivists and hospitalists of any variety of background and training, is a growing practice in a number of neurosurgical ICUs. Why is it more palatable to have these afore mentioned generecists who are neither surgeons nor neurosurgeons, rather than a credentialed Acute Care Surgeon that is fellowship trained specifically in critical care and trauma, provide critical care of non-operative as well as pre and post operative cases? The need to assure a close and continuous presence of critical care providers in trauma and neurosurgery ICUs will only increase with the Leapfrog initiative [25]. How does neurosurgery as a discipline propose to deal with this staffing challenge and the potential for a mandated incursion of non-neurosurgeon intensivists? Will sufficient neurosurgeons of the future be qualified, capable or interested in doing their own critical care? There is at least one suggestion from a neurosurgeon proposing an alternative training and career path for that specialty which mimics the general surgery ACS concept [11]. The rationale put forth for creating this alternative path echoes that of ACS proponents. However, this has not gained wide notoriety or acclaim.

Valadka has also identified that reluctance to insert intracranial pressure monitors is the most commonly reported problem with regard to neurosurgeons' care of patients with head injuries [19]. This is also borne out by the data gleaned from the NTDB ${ }^{\circ}$ which shows a very low rate of ICP monitor use, even in patients with a GCS $<9$ [25]. This is contrary to the discipline's own AANS guidelines [26]. ICP monitoring seems to have become a misplaced focus of discussions regarding the ACS scope of training and practice vis a vis neurotrauma care. There is ample evidence that providers other than neurosurgeons can competently perform this procedure [27,28]. Military surgical experience from Iraq and Afghanistan appears contrary to the characterization being proffered by neurosurgeons [15,16]. Actual accounts from the front bear out the contention that "de facto" Acute Care Surgeons can (and do) initially assess, manage, or even do craniotomies, on head injured patients without the close presence of a neurosurgeon [29].

The strong opposition to ICP monitor insertion seems to be out of proportion to the simplicity of the issue, much less the procedure. The reasons put forth to explain it seem equally difficult to defend. This is tantamount to general surgeons protesting and obstructing neurosurgeons from performing the peritoneal portion of a VP shunt. Do neurosurgical preliminary residents truly get enough experience with "bowel surgery" at the PGY 1 or 2 level? Can a neurosurgeon handle the complication of a bowel injury, dehiscence or incisional hernia? The ATLS course teaches many equally or more invasive procedures, predominantly to non-surgeons, over only two days. However, questions of student competency and patient safety with this course, which has been credited with saving thousands of lives worldwide [30], do not seem to be an issue.

There is still the issue of managing intracranial hypertension regardless of which provider type places the monitor. The neurosurgeons contend that it should be a neurosurgeon, even though this does not appear to be the case currently [19]. Arguments have been posed that the ACS will be ill equipped to master the complexities of the data gleaned from ICP monitoring. These concerns are countered by the analogous example of the current trauma/critical care surgeon interpreting complex hemodynamic data generated by pulmonary artery catheters and other sophisticated measures of cardiopulmonary function and physiology to decide upon the need for not only cardiac, but ventilatory, renal, and cerebral interventions which have a delicate interplay. Decisions are not uncommonly made by the critical care surgeon about arrhythmia control, inotropic pharmacologic support, the potential need for a balloon pump, cardiac catheterization and cardiac surgery, hemofiltration or dialysis. These surgical intensivists are quite capable of determining when a specific consultation is needed from other specialists, thereby obviating the need for their automatic involvement in care just because the heart or lung or kidney is not functioning normally. Those other specialists seem to be satisfied with this relationship.

It has been suggested that neurosurgeons do welcome partners 
from other disciplines in providing critical care services, but these are frequently neurologists. This is reportedly because of a shared interest in nervous system disease. However this points out the narrow focus of neurosurgical reasoning. Trauma is a multisystem condition, rarely limited to the nervous system alone. Only a physician who is familiar with the nuances and vagaries of the many physiologic interactions between organ systems, injuries and operations, is suitable to provide care of these challenging patients. These complex patients may, in fact, be better served by a "jack of all trades" rather than a "master" of only one.

Assuring acquisition and maintenance of competency for any scope of neurotrauma practice are legitimate concerns of both ACS proponents and neurosurgeons. Provider specific indicators of performance or competency have not been set for many areas of medicine. This is true for surgery and neurosurgery as well. In the arena of neurotrauma care, one might pose the following questions about procedures: is it 10 trauma craniotomies a year, 8 ventriculostomies, 5 Camino monitors, that assures competency? How many patients with Head-AIS (Anatomic Injury Score) greater than two must be cared for annually to maintain proficiency at neurotrauma care? The American College of Surgeons Committee on Trauma has broached this issue for surgeons that care for burn patients [31].

On the issue of monitoring and evaluating patient outcomes, what constitutes "good" vs. "bad" outcomes. Additionally, how much is related to the head injury, and how much to the neurotrauma care provider's specific management and expertise? How much is influenced by other injuries or timing and quality of rehabilitation? These types of definitions and indicators will be increasingly important in the coming era of pay for performance and a greater emphasis on maintaining competence rather than maintaining CME credits.

The competency numbers game aside, all neurosurgeons are not created equal when it comes to trauma care by virtue of varying degrees of residency exposure and current scope of practice and interest. This is much the same with general surgeons. A neurosurgeon who spends most of the time doing brain tumors and backs may not be a good neurotraumatologist, particularly for a patient with multisystem injury, similarly a general surgeon who spends most of the day doing hernias and laparoscopic cholecystectomies is not the best trauma or acute care surgeon. Not all surgeons are comfortable with their traumatology and critical care skills $[8,32]$. In fact, the phenomenon of the "traumaphile" and "traumaphobe" has been described [33].

Neurosurgeons postulate that Trauma or Acute Care Surgeons won't be able to, or want to, assume these previously exclusively neurosurgical responsibilities anyway because of a reluctance to shoulder the potential for increased liability and liability insurance premiums [16]. This may pan out to be true. However, if properly trained, credentialed, and accepted as a qualified provider of certain services to the head injured patient, they would be no more at risk than they currently are for doing bronchoscopy without being a board certified pulmonologist, or colonoscopy without being a fellowship trained colorectal surgeon or gastroenterologist, or placing a chest tube without being a board certified thoracic surgeon. The whole issue of trauma patients representing an increased medicolegal risk is more than likely myth since no objective data to support that commonly held belief exists. In fact, there is recent data to refute it [34].

The neurosurgical discipline seems particularly preoccupied with the belief that the liability issue is at the root of any perceived lack of participation in trauma care, and that limiting liability is a cure for it [16]. Efforts directed at this singular component of the problem seem to be a major focus of proposed solutions emanating from the neurosurgical leadership. Undeniably, their Liability Task Force [5] has shown the attrition rate of neurosurgeons participating in trauma care to be higher in those states categorized as liability "crisis" states. However, other information reveals that once tort reform has been established, there are a relative few who return to the ranks of trauma care [10]. More than likely this has to do with lifestyle issues and reimbursement that is not considered commensurate with effort - or to paraphrase a metaphor, "how you gonna get 'em back on the farm now that they've seen Parí".

If the medicolegal issue has its influence anywhere, it is more than likely by increasing the demand for services, regardless of what discipline is providing them. Not every head injured person need be seen by a neurosurgeon, or a trauma surgeon, or perhaps even an emergency physician. However this has become an unrealistic societal expectation, if not a standard of care. Primary care physicians often feel put upon to involve such higher-level providers either due to direct request of the patient or patient's family; or for fear of litigation if they do not, and then there is a subsequent perceived adverse outcome. There is sufficient data to support that many head injuries can be managed without ICU observation, multiple CT scans or even neurosurgical or trauma surgeon consultations [2]. Likewise, declaration of brain death or unsurvivable brain injury does not necessitate the immediate presence and intimate involvement of a neurosurgeon to the degree that it does currently. This is well within the purview of neurologists and well trained Trauma or Acute Care Surgeons [35,2].

Another prerequisite to resolving the differences between neurosurgeons and trauma surgeons on patient care issues is a shift away from a provider or organ system centered focus which is protective and defensive, to one which is centered on patient needs and services which is proactive and not self serving. Examples of shared or overlapping provision of the same patient service by different disciplines already exist and are growing. Radiologists, vascular surgeons and general surgeons are placing IVC filters; head and neck (ENT) surgeons as well as general surgeons do tracheostomies; cardiovascular surgeons and a large number of general surgeons do non-cardiac thoracic surgery (particularly in trauma); gastroenterologists along with general surgeons are doing percutaneous endoscopic gastrostomies; radiologists, surgeons, obstetricians and emergency medicine physicians perform ultrasound imaging; neurosurgeons, orthopedic surgeons (and even chiropractors), treat conditions of the spine; and on, and on. All of this with the only requisite being the imprimatur of the hospital credentialing body.

The concept of an Acute Care Surgeon is grounded in the philosophy of disease based care centered around patient needs not provider pedigree and parochialism. There is precedent for this. This same philosophy has spawned the popular new medical specialties of Emergency Medicine and Family Medicine. They were conceived on the basis of an overly burdensome volume of patients needing care and care based on the constellation of conditions occurring in a certain class of patient types. These specialties might also have been initially viewed as only occasionally dabbling in obstetrics, cardiology, orthopedics, or pediatrics, and even neurosurgery, among others. Procedures such as vaginal deliveries, c-sections, endotracheal intubation, pulmonary artery catheterization, suture of wounds, as well as non procedural skills such as primary management of stroke, acute and chronic cardiac disease, diabetes, etc. are part and parcel of these new disciplines' 
training and practice. Differing from Neurosurgery, it seems none of these "parent" governing Boards of the "true" disciplines or their professional organizations overwhelmingly protest the work of these allied disciplines. In fact, there is tolerance and even support. One can only ask, is the brain any more sacrosanct than the heart, or a baby's life?

This negative neurosurgical reaction to an ACS assuming some functions previously in the exclusive domain of a neurosurgeon brings a curious sense of déjà vu. Initially, a similar degree of intense concern and objection was voiced by radiologists in response to the thought of trauma surgeons and emergency medicine physicians performing ultrasound exams on acutely injured patients. The same arguments regarding patient safety, quality, and adequate training were raised. This equally contentious situation disappeared rather rapidly once the realities and practicalities of optimal and efficient patient care were acknowledged. Ultrasound performed on trauma patients (FAST) by non-radiologists, after close scrutiny and evaluation, has not proven to be the evil it was initially portrayed as [36]. It is now a standard practice nationally, with short courses in its performance offered to the masses [37]. This practice pattern is currently accepted, and even welcomed, by radiologists.

In large part, these interdisciplinary differences in opinion and policy relating to FAST were resolved at the local level by the practitioners who know their individual environments best. Perhaps this endorses the old adage that "all politics is local" and is a bellwether for the issues pertaining to neurotrauma care provided by an ACS.

So there are multiple examples of collaboration and facilitation of cross-disciplinary care for trauma as well as other conditions. These success stories are more than likely based on a bipartisan willingness to explore and be integrally involved in curriculum development, standard setting and change for the good of the patient and the profession, rather than resistance or outright condemnation of the whole concept without a fair hearing. A reasonable compromise and collaboration with neurosurgeons on both procedural and nonprocedural issues can certainly be found. There seems to have been a better relationship between neurosurgery and orthopedic surgery with regard to multidisciplinary spine surgery training and practice development.

A ray of hope on the horizon rests in the reaction of pediatric surgery to the Acute Care Surgery movement. In contradistinction to the neurosurgeons, pediatric surgery and it's leadership have actively lobbied for, and are seeking to facilitate, a greater exposure to that discipline in the Acute Care Surgery curriculum and are exploring avenues to have it be a required, rather than an elective, clinical component of the fellowship. This acknowledges and attempts to address the same supply, demand and societal need issues faced by neurosurgery in a more proactive, positive and collaborative fashion. This strategy will, perhaps, give the discipline of Pediatric Surgery more control of its destiny rather than less.

Broad training of an ACS which includes limited neurosurgical procedural skills, rather than a restriction of training to non procedural skills and brain resuscitation, will afford the most flexibility in addressing local needs in different catchment areas. In some Acute Care Surgeons' practices, neurosurgical skills will never need to be utilized because expeditious transfer is practical or because local neurosurgeons are committed to competently caring for trauma patients at the community hospital. In others, however, utilization of these skills before, or instead of, transfer may be crucial. From a personal practice perspective, the
ACS concept enables those who wish to, or need to, broaden their scope of practice to do so. From the standpoint of a new discipline, this concept and degree of breadth may also serve to attract more recruits to the ranks of Acute Care Surgery.

In summary then, the under supply and maldistribution of neurosurgeons coupled with the relinquishment of trauma care by a large number of rank and file neurosurgeons, and perhaps an over demand for their services, has created significant concern for access to neurotrauma care across the country. There is evidence to support that the immediate availability of a neurosurgeon to participate in the care of all trauma patients, including those with documented head injury, may not be essential to providing optimal care. Given the volume, nature, and timeliness of head injury and its care, it appears this crisis can be resolved to a great extent by having trauma surgeons who are properly trained, credentialed, and monitored, assume in-patient neurotrauma care short of formal craniotomy when hospital admission is actually indicated.

While part of the solution lies in increased supply of neurotrauma services, regardless of provider type, a second component rests in decreasing demand for these services in cases of mild as well as extremely severe head injury. Such a solution seems feasible and advantageous in a number of respects and should be seriously considered by healthcare policy makers, trauma system planners, and the leaders of the Neurosurgical and Trauma Surgery/Acute Care Surgery disciplines.

So the issues loom much larger than just who can or cannot place ICP monitors, burr holes and do craniotomies, or whether liability issues get resolved. Yet it appears the neurosurgical leadership has predominantly chosen to take this limited view of the situation. With regard to the concept of an Acute Care Surgeon and neurotrauma, their stance seems to convey more of a "can't do" rather than a "can do" attitude

Perhaps the issues revolve not so much around those who are practicing in the here and now, but around those who will come after us. Many of the arguments against an expanded scope of practice for the general trauma surgeon are mired in the "pro tem" culture and practices. It is not clear as to whether the neurosurgical leadership has taken a barometer reading on what future trainees desire in scope of practice (i.e. neurotrauma) and lifestyle? These are the questions general and trauma surgeons have asked, had answered, and now are responding to with a plan $[8,17,14,4,18,38,39]$. Perhaps a similar process of introspection and strategic planning by neurosurgeons would benefit that discipline, as well as the health care system.

This topic of Acute Care Surgery and its role as a specialty crossing traditional disciplinary lines, including those of neurosurgery, has struck a nerve with many on both sides of the issue. Perhaps the root of the pain stems not from the issue of neurotrauma care, but rather from the suggestion of change [24]. Change takes time. Change won't, and can't, happen tomorrow. No one is proposing that it should. While current trauma surgeons are not formally trained in neurotrauma care and procedures, the Acute Care Surgeon of the future can be, and perhaps needs to be. Certainly a transitional training curriculum and process must also be developed. This should be tailored to current trauma surgeons who may want to assume this broader scope of practice. Overlapping or shared responsibility for neurotrauma care can't happen tomorrow. That doesn't mean it shouldn't be a mutual goal, and that objective, realistic, and open minded dialogue between disciplines can't start today.

We have recently started to hear tell of the "House of Surgery" as 
a result of the American College of Surgeons' initiatives to research and resolve a perceived crisis in access to emergency surgical care of all types [12]. Getting the "House" in order begins one room at a time. It would seem to serve little purpose quibbling over who should paint the trauma room which color, while the people who need it suffer from exposure to the elements without benefit of access to shelter. There are many painters that can share the job or be apprenticed, if they are allowed to. Let's face it, you don't always need a muralist to just paint a wall or a plasterer to do the spackling before a painter paints! An elitist or trade union mentality does not serve our profession or our patients well.

There is, and needs to be, a greater role for the current trauma surgeon than just brain resuscitation and patient stabilization. Trauma surgeons are already well schooled and well experienced in these skills. Trauma surgeons require little, if any, assistance or education from the neurosurgeon in this limited area, yet this is the only offer of collaboration on curriculum development and training that has been readily forthcoming. What would be more constructive and productive for neurosurgery, or any other discipline (orthopedics, thoracic surgery, pediatric surgery, gynecology, etc.) concerned about the impact of the acute care surgery initiative on their patients and practices, might be to articulate the following as it applies to their specialty:

- Confirm or credibly rebut the claim that a void in neurotrauma care currently exists, and stands to worsen, by presenting its own current demographic evaluation and needs assessment.

- Define the specific services required to treat the spectrum of head injured patients from mild to severe and unsurviveable (perhaps a restatement or reformatting of the AANS guidelines - similar to the American College of Surgeons' optimal resources document )

- Propose how neurosurgery plans to realistically meet patient needs and provide those services consistently, efficiently and effectively across the country given the broad spectrum of services and the patient, as well as neurosurgeon, demographics.

- Provide a clear definition of the head injury patient population for which the board certified neurosurgeon is the primary provider/consultant of choice.

- Propose a feasible means to get those patients to the provider of choice (i.e. a systems approach-- triage criteria/system or regionalization scheme, etc).

- Elucidate which other providers (if any) are seen as available, suitable, and willing to share the responsibility and burden of neurotrauma care if neurosurgeons alone cannot adequately meet the demonstrated needs.

- Propose how one trains and credentials these other providers.

- Set standards for neurotrauma care that will apply to all provider's regardless disciplinary background along with proposals for how the performance of all providers should be monitored.

- Define the minimum number of a particular invasive procedure (decompressive procedure, ICP monitor placement, etc.) per year are required to maintain competency for any neurotrauma care provider (including neurosurgeons).

- Define the minimum number of seriously head injured patients
(AIS $>2$ ) managed operatively and non-operatively annually that is required to demonstrate proficiency at neurotrauma care for any provider (including neurosurgeons).

- Verify, and propose potential solutions to, the over demand for neurotrauma services regardless of provider.

- Propose a plan that will obviate the overburdening of resources at the regional referral centers and of the neurosurgeons who staff them.

- Propose a plan to address lifestyle as well as liability issues which impede neurosurgical participation in trauma care.

In the final analysis, it's not about pedigree or turf. It's about patients and patient needs. It's about having committed, capable, care providers with common sense, who put patient interests before parochial practices rooted in tradition, and whose performance is well monitored. In some communities and hospitals that provider will be a Neurosurgeon. In some, perhaps, an Acute Care Surgeon. In others an Emergency Medicine practitioner or physician extender... and in many, conceivably, just somebody's mom.

\section{Disclaimer}

The views expressed in this article are solely those of the author and do not necessarily represent the position of any professional organizations with which he is affiliated.

\section{References}

1. Bonnie RJ, Fulco CE, Liverman CT (1999) Reducing the Burden of Injury Advancing Prevention and Treatment. Institute of Medicine, National Academy Press, Washington, D.C

2. Esposito TJ, Reed RL 2nd, Gamelli RL, Luchette FA (2005) Neurosurgical coverage: essential, desired, or irrelevant for good patient care and trauma center status. Ann Surg 242: 364-370.

3. (2001) Injury Prevention and Control: Injury Fact Book. Centers for Disease Control and Prevention.

4. Esposito TJ, Rotondo M, Barie PS, Reilly P, Pasquale MD (2006) Making the case for a paradigm shift in trauma surgery. J Am Coll Surg 202: 655-667.

5. Society of Neurological Surgeons Liability Insurance Task Force: Survey of Academic Neurosurgical Programs. Unpublished data

6. Griffen FD (2007) A perfect storm: ED crisis and the on-call surgeon. J Fam Pract 63: 73-76.

7. Valadka AB (2004) The ER - Who is Answering the Call? In Some Hospitals, Not Neurosurgeons. Bull AANS 13: 6-12.

8. Esposito TJ, Kuby AM, Unfred C, et al. (1993) National Survey of Surgeons on Trauma Care Issues: Robert Wood Johnson Foundation Report. Newark, NJ.

9. Esposito TJ, Crandall M, Reed RL, Gamelli RL, Luchette FA (2006) Socioeconomic factors, medicolegal issues, and trauma patient transfer trends: Is there a connection? J Trauma 61: 1380-1386.

10. Byrne RW, Bagan BT, Slavin KV, Curry D, Koski TR, et al. (2008) Neurosurgical emergency transfers to academic centers in Cook County: a prospective multicenter study. Neurosurgery 62: 709-716.

11. Origitano TC (2005) On the Issue of Neurosurgical Transfers to Academic Medical Centers. Assn Neurol Surg Bull 14: 12-13.

12. Division of Advocacy and Health Policy (2006) A growing crisis in patient access to emergency surgical care. Bull Am Coll Surg 91: 8-19.

13. (2006) Institute of Medicine: Future of Emergency Care Series. Hospital Based Emergency Care - At the Breaking Point. National Academy Press Washington, D.C.

14. Rotondo MF, Esposito TJ, Reilly PM, Barie PS, Meredith JW, et al. (2005) The position of the Eastern Association for the Surgery of Trauma on the future of trauma surgery. J Trauma 59: 77-79. 
Citation: Esposito TJ (2012) Neurosurgeons, Acute Care Surgeons or Moms: Who Should Care for the Head Injured? J Trauma Treat 1:137. doi:10.4172/2167-1222.1000137

Page 7 of 7

15. Rotondo MF, Esposito TJ, Reilly PM, Barie PS, Meredith JW, et al. (2005) The position of the Eastern Association for the Surgery of Trauma on the future of trauma surgery. J Trauma 59: 77-79.

16. Valadka AB, Timmons SD, Ellenbogen RG (2006) Delivery of emergency neurosurgical care. J Am Coll Surg 203: 962-966.

17. Esposito TJ, Leon L, Jurkovich GJ (2006) The shape of things to come: results from a national survey of trauma surgeons on issues concerning their future. $J$ Trauma 60: 8-16

18. Esposito TJ (2006) Rank and file weighs in on trauma and general surgery issues: results frow a survey of ACS Fellows. Bull Am Coll Surg 91: 13-20.

19. Valadka AB, Andrews BT, Bullock MR (2001) How well do neurosurgeons care for trauma patients? A survey of the membership of the American Association for the Surgery of Trauma. Neurosurgery 48: $24-25$.

20. Benzil DL (2005) Patient Transfers Spark Town-Gown Tension: AMC Directors See Pros and Cons for Training Programs, Patients. AANS Bull 14: 24-25.

21. Trunkey DD (2003) What Price Commitment. Bull Am Coll Surg 88: 8-16.

22. Wester T, Fevang LT, Wester K (1999) Decompressive surgery in acute head injuries: where should it be performed? J Trauma 46: 914-919.

23. Rinker CF, McMurry FG, Groeneweg VR, Bahnson FF, Banks KL, (1998) Emergency craniotomy in a rural Level III trauma center. J Trauma 44: 984-989.

24. Esposito TJ (2007) Moving the cheese: a commentary on debate over the acute care surgery initiative. Surgery 142: 414-419.

25. Birkmeyer JD, Dimick JB (2004) The Leapfrog Group's Patient Safety Practices, 2003: The Potential Benefits of Universal Adoption.

26. Chesnut RM (1997) Guidelines for the management of severe head injury: what we know and what we think we know. J Trauma 42: 19S-22S.

27. Kaups KL, Parks SN, Morris CL (1998) Intracranial pressure monitor placement by midlevel practitioners. J Trauma 45: $884-886$.
28. Ko K Conforti A (2003) Training protocol for intracranial pressure monitor placement by non-neurosurgeons: 5 year experience. J Trauma 55: 480-483.

29. Schwab CW (2007) Crises and War: Stepping Stones to the Future. J Trauma 62: 1-16.

30. http://www.worldcat.org/title/atls-advanced-trauma-life-support-program-fordoctors/oclc/60551494?tab=details

31. (2006) Committee on Trauma, American College of Surgeons: Resources for Optimal Care of the Injured Patient 2006. American College of Surgeons, Chicago, IL.

32. Esposito TJ, Maier RV, Rivara FP, Carrico CJ (1991) A statewide profile of general surgery trauma practice. J Trauma 31: 39-42.

33. Esposito TJ, Kuby AM, Unfred C, Young HL, Gamelli RL (1994) Perception of differences between trauma care and other surgical emergencies: results from a national survey of surgeons. J Trauma 37: 996-1002.

34. Stewart RM, Johnston J, Geoghegan K, Anthony T, Myers JG, et al. (2005) Trauma surgery malpractice risk: perception versus reality. Ann Surg 241: 969975.

35. Esposito TJ, Luchette FA, Gamelli RL (2006) Do we need neurosurgical coverage in the trauma center? Adv Surg 40: 213-221.

36. Fernandez L, McKenney MG, McKenney KL, Cohn SM, Feinstein A, et al (1998) Ultrasound in blunt abdominal trauma. J Trauma 45: 841-848.

37. Knudson MM (2002) Ultrasound in Emergency Medicine and Trauma. J Trauma 52: 603-604.

38. Shackford S (2006) How then shall we change? J Trauma 60: 1-7.

39. Committee to Develop the Reorganized Specialty of Trauma, Surgical Critical Care, and Emergency Surgery (2005) Acute care surgery: trauma, critical care, and emergency surgery. 58: 614-616. 